\title{
Comparative proteomic analysis of saliva from dogs with and without obesity-related metabolic dysfuntion
}

\author{
Sónia Lucena ${ }^{\mathrm{a}, \mathrm{b}}$, Ana Varela Coelho ${ }^{\mathrm{c}}$, Sandra I. Anjo ${ }^{\mathrm{d}, \mathrm{e}}$, Bruno Manadas ${ }^{\mathrm{d}}$, Vladimir Mrljak ${ }^{\mathrm{f}}$, \\ Fernando Capela e Silva ${ }^{\mathrm{a}, \mathrm{g}}$, Elsa Lamy ${ }^{\mathrm{a}}$, Asta Tvarijonaviciute $\mathrm{e}^{\mathrm{h}, *}$ \\ a Institute of Agrarian and Environmental Environmental Sciences (ICAAM), University of Évora, Portugal \\ ${ }^{\mathrm{b}}$ Department of Veterinary Medicine, School of Science and Technology, University of Évora, Portugal \\ ${ }^{\mathrm{c}}$ Institute of Chemical and Biological Technology (ITQB), New University of Lisbon, Portugal \\ ${ }^{\mathrm{d}}$ CNC - Center for Neuroscience and Cell Biology, University of Coimbra, Portugal \\ ${ }^{\mathrm{e}}$ Faculty of Medicine, University of Coimbra, Portugal \\ ${ }^{\mathrm{f}}$ Clinic for Internal Dieaases, Faculty of Veterinary Medicine, University of Zagreb, Heinzelova 55, 10000 Zagreb, Croatia \\ ${ }^{\mathrm{g}}$ Department of Biology, School of Science and Technology, University of Évora, Portugal \\ ${ }^{\mathrm{h}}$ Interlab-UMU, Regional Campus of International Excellence Campus Mare Nostrum, University of Murcia, Murcia 30100, Spain
}

\section{A R T I C L E I N F O}

\section{Keywords:}

Dog

Saliva

Proteome

Obesity

Metabolic syndrome

\begin{abstract}
A B S T R A C T
Dogs develop only some of the components of the human metabolic syndrome (MetS). Thus, in order to study possible MetS-related alterations in dogs, human MetS criteria were adapted to define canine MetS or so-called obesity-related metabolic dysfunction (ORMD). The main objective of this study was to identify changes in the salivary proteome of obese dogs with ORMD in comparison with obese dogs without ORMD which may constitute potential salivary biomarkers for assessing ORMD. In a first phase, 12 adult obese dogs with ORMD $(\mathrm{N}=6)$ and without ORMD $(\mathrm{N}=6)$ were included in the study. Subsequently, and with the aim of validating and strengthening the results, additional 12 obese dogs (6 with and 6 without ORMD) were tested in an independent experiment following the same protocol. Saliva samples were subjected to a quantitative proteomics analysis and the levels of nine salivary proteins were found to be significantly different between groups, among them those which had greatest fold-change were proteins involved in glycolysis and oxidative stress. In conclusion, despite metabolic syndrome to include different combinations of diseases, the observation of differences in salivary proteome suggests a potential of this fluid to understand the pathophysiology of the disease. Significance: This is the first study evaluating proteomes of saliva in dogs, as a non invasive sample, in order to increase knowledge about the metabolic/physiopathological changes related to obesity-related metabolic dysfunction (ORMD) together with the identification of potential biomarkers for its diagnosis. As approximately $20 \%$ of dogs with naturally occurring obesity were described to suffer ORMD associated with insulin resistance and hypoadiponectinemia, the fact that indicate possible links between ORMD and associated diseases.
\end{abstract}

\section{Introduction}

In humans, the term 'metabolic syndrome' (MetS) describes a clustering of obesity (especially visceral obesity), hypertension, insulin resistance or hyperglycaemia, and hyperlipidaemia, especially increased fasting triglycerides and decreased High-Density Lipoprotein Cholesterol (HDL-C) [1]. The MetS itself causes no clinical signs, but comprises a set of important risk factors for diseases that cause morbidity and mortality in humans, including non-alcholic liver disease, cardiovacuscular disease, stroke, and type 2 diabetes [2]. Dogs develop some of the components of the human MetS: obesity [3], insulin resistance [4], increased blood pressure [5], and hyperlipidaemia [6]. Nevertheless, although liver alterations in canine MetS were reported [7], the most important consequences of the MetS in humans such as type 2 diabetes (T2D), stroke and coronary heart disease, either do not exist or are very rare in dogs suggesting that dogs could have protective mechanisms or that dogs lack pathophysiological elements present in humans [8]. In order to study possible MetS-related alterations in dogs, human MetS criteria were adapted to define canine MetS or so-called obesity-related metabolic dysfunction (ORMD) [9]. Approximately $20 \%$

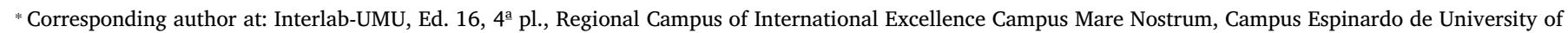
Murcia, 30100, Espinardo, Murcia, Spain.

E-mail address: asta@um.es (A. Tvarijonaviciute). 
of dogs with naturally occurring obesity were described to suffer ORMD [9] associated with insulin resistance and hypoadiponectinemia [9-11].

Saliva has gained interest for biomarker identification, mainly due to the non-invasive nature of its collection, at the same time that it contains glandular and blood-born molecules whose levels can change under different conditions [12]. In humans, various MetS biomarkers were found in saliva samples, such as salivary HDL-C and fasting glucose levels, adipokines (such as adiponectin, leptin, resistin) and proinflammatory markers such as C-reactive protein (CRP), insulin, ghrelin, tumour necrosis factor alpha (TNF- $\alpha$ ), interleukins, either in adults or adolescents and children [13-15]. The results of those studies provide useful information about the development of this metabolic disease and establish that saliva may be a fluid of interest in the study of this syndrome [14].

In present study, we hypothesized that dogs with metabolic dysfunction could have a different salivary protein composition comparatively to dogs without ORMD. Thus, the main objective of this study was to identify changes in the salivary proteome in obese dogs with ORMD that can help to understand the metabolic/physiopathological changes related to this condition together with the identification of potential biomarkers for its diagnosis.

\section{Materials and methods}

\subsection{Ethical note}

The study protocol was approved by local and regional Research Ethics Committees (323/2017 and A13170806, respectively).

\subsection{Animals}

A total of 24 adult dogs of different breeds were included in this study. Saliva samples from six castrated adult obese dogs without ORMD (non-ORMD group) and six castrated adult obese dogs with ORMD (ORMD group) were used for the proteomic screening while the remaining animals (six with and six without ORMD) were further used to validate data obtained in the initial screening, as detailed in Section 2.7. All animals were client-owned dogs brought for routine check-up or vaccination to different private veterinary clinics of Murcia Region, Spain. All dogs were evaluated for their general health status and only those that did not present signs of other than obesity diseases, as based on physical examination, CBC and biochemistry analyses. In addition, CRP was measured in all dogs in order to discard active inflammation; only dogs with CRP $<12 \mathrm{mg} / \mathrm{L}$ were considered for the inclusion in the study. Body condition score (BCS) was determined using a validated 5 -point body condition score system (were 1 means very thin, 2 - underweight, 3 - ideal weight, 4 - overweight, 5 - obese) in both groups [16]. The descriptive clinical data of dogs from both groups are detailed in Table 1.

\subsection{Definition of obesity-related metabolic dysfunction}

Dogs were considered as having ORMD if met previously described criteria [9]: (a) BCS 4-5/5; and (b) any two of the following: 1) plasma triglycerides $>200 \mathrm{mg} / \mathrm{dL}$; 2) plasma cholesterol $>300 \mathrm{mg} / \mathrm{dL}$; 3) systolic arterial presure (SAP) $>160 \mathrm{mmHg}$; 4) fasting plasma glucose $>100 \mathrm{mg} / \mathrm{dL}$, or previously diagnosed diabetes mellitus [9].

Blood pressure in all dogs was measured non-invasively using an oscillometric method. All dogs were fully conscious. A cuff of the appropriate size (e.g. the cuff chosen had a width of $\sim 40 \%$ circumference of the leg) was placed on the right forelimb. Once the dog was calm and still, at least five systolic arterial pressure (SAP) readings were taken and averaged.

\subsection{Serum analysis}

Serum total cholesterol, triglycerides and glucose were measured in an automated biochemistry analyser (Olympus AU600, Beckman Coulter, Brea, USA) using commercially available reagents and following the instructions of the manufacturer.

\subsection{Saliva analysis}

\subsubsection{Saliva collection}

Saliva samples were collected as previously described [17]. A sponge was placed in each dog's mouth, left in contact with the cheek mucosa for 1-2 min, and was then placed into the Salivette device for centrifugation $\left(3000 \mathrm{~g}, 10 \mathrm{~min}, 4^{\circ} \mathrm{C}\right)$. After centrifugation, saliva was transferred to $1.5 \mathrm{~mL}$ polyethylene tubes and stored at $-80^{\circ} \mathrm{C}$ until analysis.

\subsubsection{Total protein concentration}

Bradford method protein assay with BSA as the standard protein (Pierce Biotechnology, Rockford, IL, USA) was performed to determine the total protein concentration of each sample. Standards and samples were run in triplicate, in 96 wells microplates. Absorbance was read at $600 \mathrm{~nm}$ in a microplate reader (Glomax, Promega).

\subsubsection{Protein digestion in solution}

The volume of each saliva sample correspondent to a total of $50 \mu \mathrm{g}$ of protein was added to $10 \mu \mathrm{L}$ of $6 \mathrm{M}$ Urea $50 \mathrm{mM}$ ammonium bicarbonate (AB). Then $1 \mu \mathrm{L}$ of $\mathrm{NaOH} 0.5 \mathrm{M}$ was also added to adjust $\mathrm{pH}$ to 8-8.5. To perform the reduction $1.43 \mu \mathrm{L}$ of dithiothreitol (DTT) $700 \mathrm{mM}$ was added and the samples incubated for $1 \mathrm{~h}$ at room temperature. Then alkylation was made adding $4.29 \mu \mathrm{L}$ of iodoacetamide (IAA) $700 \mathrm{mM}$, with a posterior incubation of $30 \mathrm{~min}$ at room

Table 1

The baseline characteristics of dogs included in salivary proteomic and validation studies with and without obesity-related metabolic dysfunction (ORMD).

\begin{tabular}{|c|c|c|c|c|c|c|}
\hline \multirow[t]{2}{*}{ Variable } & \multicolumn{3}{|c|}{ Animals included in proteomic analysis } & \multicolumn{3}{|c|}{ Animals included in validation } \\
\hline & Non-ORMD & ORMD & $\mathrm{P}$ & Non-ORMD & ORMD & $\mathrm{P}$ \\
\hline Breed & $\begin{array}{l}\text { German Shepherd, } \\
\text { Mongrel }\end{array}$ & $\begin{array}{l}\text { Beagle, Mongrel, Shih Tzu, Scotish } \\
\text { terrier, Cocker spaniel }\end{array}$ & & $\begin{array}{l}\text { Mongrel (5), Podenco } \\
\text { Ibicenco }\end{array}$ & $\begin{array}{l}\text { Mongrel (2), Beagle, Scottish terrier, } \\
\text { Golden retriever, Boxer }\end{array}$ & \\
\hline Age, years & $6.8(2.5-11.0)$ & $8.0(3.0-11.6)$ & 0.228 & $5.9(1.8-9.1)$ & $6.5(2.8-10.4)$ & 0.560 \\
\hline Sex & 3 females and 3 males & 3 females and 3 males & & 4 females and 2 males & 3 females and 3 males & \\
\hline $\begin{array}{l}\text { Body condition score, } 5 \text { - point } \\
\text { scale }\end{array}$ & $4-5$ & 5 & & $4-5$ & $4-5$ & \\
\hline Body Weight, kg & $15.8(8.9-24.7)$ & $12.0(8.7-18.7)$ & 0.254 & $25.8(21.0-30.7)$ & $18.0(9.5-34.3)$ & 0.571 \\
\hline Total cholesterol, mg/dL & 244.5(209-649) & $381(269-433)$ & 0.119 & $273.0(195.8-283.5)$ & $344.5(319.8-373.2)$ & 0.002 \\
\hline Triglycerides, $\mathrm{mg} / \mathrm{dL}$ & $83.5(66.0-96.0)$ & $88.0(69.7-355.0)$ & 0.333 & $88.0(64.5-103.0)$ & $90.0(49.1-152.1)$ & 0.898 \\
\hline Glucose, $\mathrm{mg} / \mathrm{dL}$ & $94.0(82.0-144.0)$ & $107.5(90.0-111.0)$ & 0.083 & $84.5(82.5-99.8)$ & $104.6(101.1-108.6)$ & 0.009 \\
\hline $\begin{array}{l}\text { Systolic arterial pressure } \\
\text { (SAP) }\end{array}$ & $140.9(137.3-157.5)$ & $139.8(94.5-149.0)$ & 0.329 & $133.2(126.3-137.2)$ & $144.8(134.4-161.4)$ & 0.127 \\
\hline
\end{tabular}


temperature in the dark. To quench the excess of IAA $7.5 \mu \mathrm{L}$ of 500 mMN-acetyl cysteine (NAC) was added to the samples and incubated for $15 \mathrm{~min}$ at room temperature. A volume of $486.8 \mu \mathrm{L}$ of $\mathrm{AB} 50 \mathrm{mM}$ was added to samples to dilute the urea concentration to $1 \mathrm{M}$. Immediately before trypsin digestion, $2 \mu \mathrm{g}$ of an internal standard (MBP-GFP fusion protein - Maltose-binding protein combined with Green Fluorescent Protein) was added. The digestion with $5 \mu \mathrm{L}$ of trypsin (stock $1 \mu \mathrm{g} / \mathrm{uL}$ ) added to each sample was done for $18 \mathrm{~h}$ at $37^{\circ} \mathrm{C}$. To stop digestion a volume of $3 \mu \mathrm{L}$ of formic acid (FA) was added to each sample. Subsequently, a cleaning/concentration step was performed using OMIX C18 tips (Agilent Technologies), according to manufacturer recommendations, by passing the mixture through the tips and eluting the peptides by adding $70 \%$ ACN in $0.1 \%$ FA solution. The flow was transferred to a new $1.5 \mathrm{~mL}$ polyethylene tube and the mixture dried using a speed vac (LabConco, CentriVap micro IR).

\subsubsection{SWATH-MS analysis - data acquisition}

Saliva samples were analysed on a TripleTOF ${ }^{\mathrm{TM}} 6600$ System $\left(\mathrm{Sciex}^{\circledast}\right)$ using information-dependent acquisition (IDA) of pooled samples for protein identification and SWATH-MS acquisition of each individual sample for protein quantification [18]. Peptides were resolved by liquid chromatography (nanoLC Ultra 2D, Eksigent ${ }^{\circledR}$ ) on a MicroLC column Halo Fuse Core ${ }^{\mathrm{Tm}}(300 \mu \mathrm{m}$ ID $\times 15 \mathrm{~cm}$ length, $2.7 \mu \mathrm{m}$ particles, $90 \AA$ pore size, Eksigent ${ }^{\circledast}$ ) at $5 \mu \mathrm{L} / \mathrm{min}$ with 45 min linear gradient from $5 \%$ to $30 \%$ of ACN in $0.1 \%$ FA and $5 \%$ dimethylsulfoxide (DMSO). Peptides were eluted into the mass spectrometer using an electrospray ionization source (DuoSpray ${ }^{\mathrm{TM}}$ Source, ABSciex $^{\circledast}$ ) with a $50 \mu \mathrm{m}$ internal diameter (ID) stainless steel emitter (NewObjective). For IDA experiments, the mass spectrometer was set to scanning full spectra $(\mathrm{m} / \mathrm{z} 350-1250)$ for $250 \mathrm{~ms}$, followed by up to $100 \mathrm{MS} / \mathrm{MS}$ scans $(\mathrm{m} / \mathrm{z}$ 100-1500 from a dynamic accumulation time - minimum $30 \mathrm{~ms}$ for precursor above the intensity threshold of 2000 - in order to maintain a cycle time of $3.3 \mathrm{~s}$ ). Candidate ions with a charge state between +2 and +5 and counts above a minimum threshold of 10 counts per second were isolated for fragmentation and one MS/MS spectra was collected before adding those ions to the exclusion list for $15 \mathrm{~s}$ (mass spectrometer operated by Analyst ${ }^{\circledR}$ TF 1.7, Sciex ${ }^{\circledast}$ ). Rolling collision was used with a collision energy spread of 5. For SWATH-MS based experiments, the mass spectrometer was operated in a looped product ion mode [19] and the same chromatographic conditions used as in the IDA run described above. A set of 168 windows of variable width (containing an $\mathrm{m} / \mathrm{z}$ of 1 for the window overlap) was constructed covering the precursor mass range of $m / z \quad 350-1250$. A $50 \mathrm{~ms}$ survey scan $(\mathrm{m} / \mathrm{z}$ 350-1250) was acquired at the beginning of each cycle for instrument calibration and SWATH MS/MS spectra were collected from $\mathrm{m} / \mathrm{z}$ $100-1500$ for $20 \mathrm{~ms}$ resulting in a cycle time of $3.29 \mathrm{~s}$ from the precursors ranging from $m / z 350$ to 1250 . The collision energy (CE) applied to each $m / z$ window was determined considering the appropriate $\mathrm{CE}$ for a +2 ion centred upon this window and the collision energy spread (CES) was also adapted to each $\mathrm{m} / \mathrm{z}$ window.

\subsubsection{SWATH-MS data analysis - protein identification and quantification}

A specific library of precursor masses and fragment ions was created by combining all files from the IDA experiments (one pool per group of samples), and used for subsequent SWATH processing.

Peptide identification and library generation were performed with ProteinPilot software (v5.0, Sciex ${ }^{\circledR}$ ), using the following parameters: i) search against a database composed by the Uniprot's reference proteome UP000002254 from Canis lupus familiaris (85,188 entries, release at December 2017) and MBP-GFP; ii) iodoacetamide alkylated cysteines as fixed modification; iii) trypsin as digestion type. An independent False Discovery Rate (FDR) analysis, using the target-decoy approach provided by ProteinPilot ${ }^{\mathrm{Tm}}$, was used to assess the quality of the identifications and confident identifications were considered when identified peptides and proteins reached a 5\% local FDR [20,21]. Data processing was performed using SWATH $^{\mathrm{TM}}$ processing plug-in for
PeakView $^{\mathrm{TM}}$ (v2.0.01, AB Sciex $\left.{ }^{\circledast}\right)$. After retention time adjustment using the MBP-GFP peptides, up to 15 peptides, with up to 5 fragments each, were chosen per protein, and quantitation was attempted for all proteins in library file that were identified from ProteinPilot ${ }^{\mathrm{TM}}$ search. Peptides' confidence threshold was determined based on a FDR analysis using the target-decoy approach and those that met the 1\% FDR threshold in at least three biological replicates were retained, and the peak areas of the target fragment ions of those peptides were extracted across the samples using an extracted-ion chromatogram (XIC) window of $4 \mathrm{~min}$ and $100 \mathrm{ppm}$ error. The levels of the proteins were estimated by summing all the filtered transitions from all the filtered peptides for a given protein and normalized to the total intensity obtained in each sample.

\subsection{BLAST Search and Gene Ontology (GO) classification}

Proteins for which differential accumulation was found by liquid chromatography-tandem mass spectrometry (LC MS/MS) analysis were submitted to functional classification by PANTHER (protein annotation through evolutionary relationship) (http://www.pantherdb.org/). Canis lupus familiaris was the selected organism. Molecular function and biological processes pie-charts were constructed.

The BLAST program downloaded from NCBI was used for annotation of "uncharacterized proteins" and to assess homologies with proteins with known functions.

\subsection{Validation of proteomic analysis}

Saliva samples from 12 overweight/obese dogs (BCS 4-5/5) were used to validate the findings from the initial proteomic analysis (Table 1). Saliva samples collection and proteomic analysis were performed as described for the first 12 dogs analysed (Sections 2.5 and 2.6). SWATH-MS analysis was used for salivary protein quantification and comparison between the dogs with and without ORMD, following the same protocol already described in Section 2.5.

\subsection{Statistical analysis}

Each variable (i.e., quantified protein) was tested for normality using the Shapiro-Wilk test. Outlier's values were removed, when existent. Univariate analysis, for comparison of protein levels between ORMD and non-ORMD groups, was performed using the Student's $t$-test or the non-parametric Mann-Whitney test, depending if the data follow a normal distribution. Statistical significance was considered for $p<0.05$ and analysis procedures were achieved using the SPSS 21.0 software package (SPSS Inc., Chicago, USA).

For Multivariate Analysis, partial least squares discriminant analysis (PLS-DA) was used taking into account the interdependence among proteins. Data normalization was performed by a pooled sample from group non-ORMD and cube root transformation was used. Discriminant variables selection was done using variable importance in the projection (VIP) with a threshold of 2.0. This was done using Metaboanalyst 4.0 [22].

For results validation, the option "Biomarker meta-analysis", in Metaboanalyst software was used, for confirmation of the proteins presenting the same differences/tendencies in both sub-populations of animals tested.

\section{Results}

In the present study, 300 proteins were identified in dog saliva (supplementary table 1). These proteins have different molecular functions, with $47.3 \%$ having catalytic activity and $37.2 \%$ being involved in binding. A lesser percentage of dog salivary proteins have diverse molecular functions, such as structural molecule activity (6.9\%), antioxidant (4.8\%), signal transducer (1.6\%), receptor $(1.1 \%)$ 


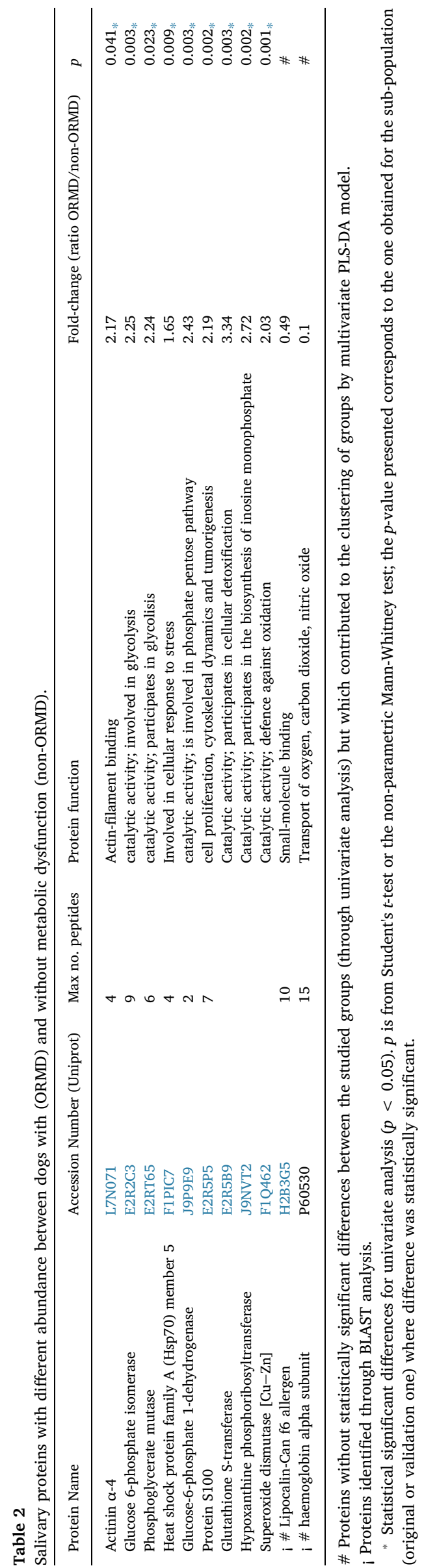

and translation regulator $(1.1 \%)$ activities. From the proteins identified, $28.3 \%$ are involved in cellular processes and $24.1 \%$ in metabolic processes (Supplementary Fig. 1). To the best of author's knowledge, 51 of the identified proteins were not previously reported in canine saliva (Supplementary Table 2).

Among the identified proteins, 257 were quantified in the initial screening (Supplementary Table 1.2) and 241 were quantified in the validation study (Supplementary Table 1.3). The amounts of these proteins were compared between ORMD and non-ORMD groups, first in the sub-population initially constituted for the study (screening) and, subsequently in the sub-population used for validation of the results. One of the animals from non-ORMD group, of the sub-population used for screening, was observed to be an outlier in the amounts of most of the salivary proteins present. Moreover, looking for serum biochemical parameters, total cholesterol reached a value higher than $600 \mathrm{mg} / \mathrm{dL}$. For these reasons, it was removed from analysis.

Univariate analysis of the quantified proteins (both from initial screening and validation sub-population) revealed nine proteins with significant differential levels between ORMD and non-ORMD groups (Table 2). These nine proteins met one of the following criteria: 1) to present statistically significant differences $(\mathrm{P}<0.05$ ), between ORMD and non-ORMD dogs, in both the screening and the validation assays; 2) to present statistically significant differences, between groups, in only one of the sub-populations, presenting a tendency $(\mathrm{P}<0.1)$ in the other (Fig. 1). These nine proteins were related to catalytic activity, some participating in carbohydrate metabolism, cellular response to stress and detoxification and with transport functions. In all cases the protein levels were increased in the ORMD group.

The multivariate PLS-DA model shows a clear separation of the groups (ORMD vs. non-ORMD), as illustrated for the sub-population used for screening (Fig. 2). The proteins that contributed to this separation (VIP $\geq 2$ ) in both sub-populations (original and validation one) were two uncharacterized proteins (J9NXL3 and F1Q3K7) (Fig. 3). BLAST analysis revealed that the identified "uncharacterized protein" (J9NXL3) presented 99.3\% homology with the haemoglobin alpha subunit from Canis lupus familiaris (P60530) and the other "uncharacterized protein" (F1Q3K7) presented 100\% homology with the lipocalin-Can f6 allergen from Canis lupus familiaris (H2B3G5).

\section{Discussion}

This is the first study evaluating the salivary proteome in obese dogs with and without obesity-related metabolic dysfunction (ORMD and non-ORMD, respectively). Initially, saliva from a sub-population of 12 dogs ( 6 with and 6 without ORMD) were analysed, with a subsequent analysis being performed in an independent sub-population of 12 different dogs ( 6 with and 6 without ORMD) for validation of results.

In the present study, $83 \%$ of the detected proteins were reported previously in canine saliva using proteomic approaches [23], while 51 identified proteins have never been reported before for dog saliva. At least in part, this can be attributed to different methodologies and dog breeds used among the diverse studies, since different salivary proteomes were described in dogs of different breeds [24,25].

Analysis in both sub-populations of dogs allowed the observation of nine salivary proteins with increased abundance in ORMD group. These proteins are involved in stress/oxidation defence [superoxide dismutase [Cu-Zn], glutathione-S-transferase, heat shock protein 70 (Hsp70)], related with the glycolytic pathways (glycolysis - glucose 6-phosphate isomerase, phosphoglycerate mutase; and pentose phosphate pathway Glucose-6-phosphate 1-dehydrogenase), cytoskeletal dynamics (protein S100, actinin $\alpha-4$ ), protein degradation (cathepsin D) and transport (lipocalin, haemoglobin alpha subunit).

Metabolic syndrome is known to be related with oxidative stress, a condition in which an imbalance results between the production and inactivation of reactive oxygen species [26]. As such, it is understandable that proteins involved in oxidative stress defence are 


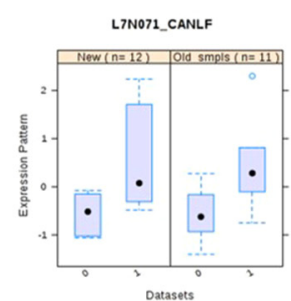

E2RSPS_CANLF

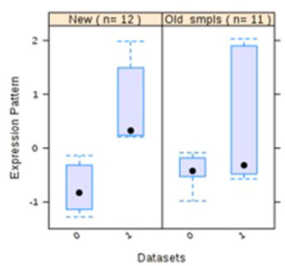

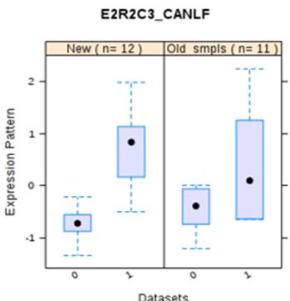

2R5B9_CANLF

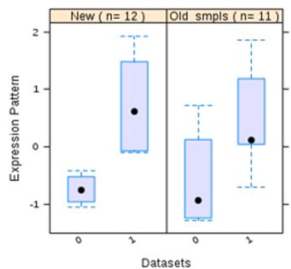

E2RT65_CANLF

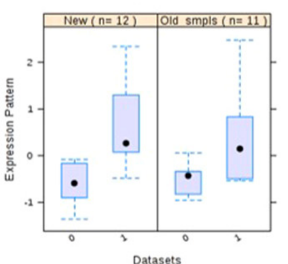

JONVT2_CANLF

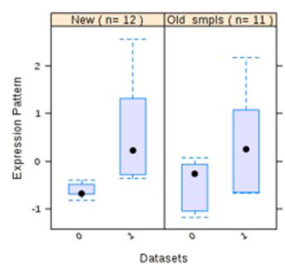

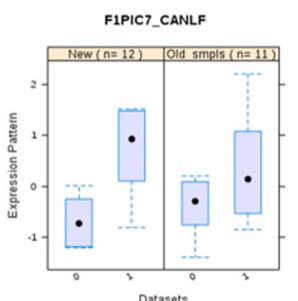

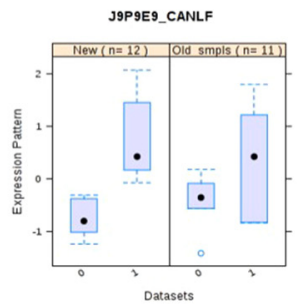

F1Q462 CANLF

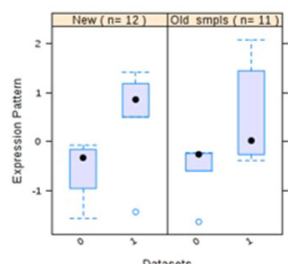

$0-$ non-ORMD 1. ORMD

Fig. 1. Salivary proteins presenting consistent variations between ORMD and non-ORMD groups in both the screening and the validation assays.

\section{Scores Plot}

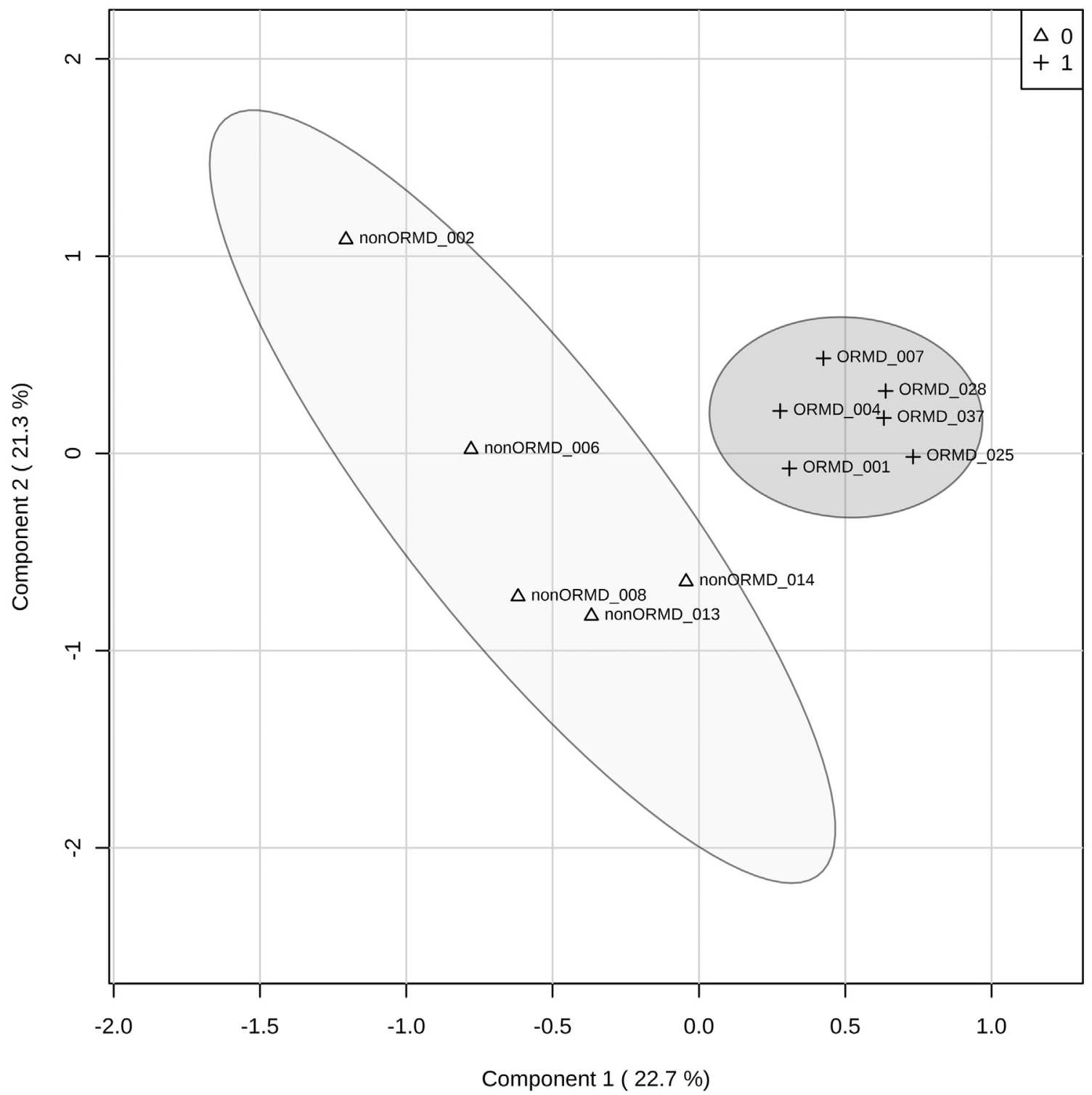

Fig. 2. Partial Least Square Determinant Analysis (PLS-DA) model for dog saliva samples of original sub-population [non-ORMD $(n=5, \Delta)$ vs. ORMD $(n=6,+)$ ]. $X$ and $\mathrm{Y}$ axis show principal component 1 (PC1) and principal component 2 (PC2), respectively, and the contribution of each of them for explaining the total variance. 
A

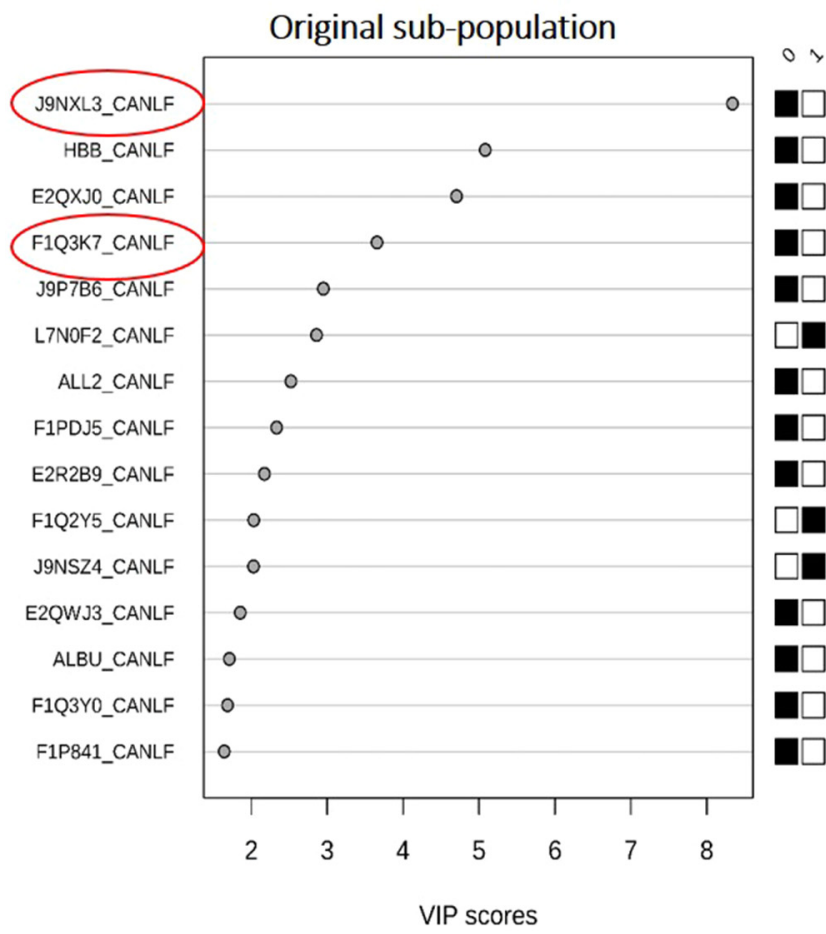

B

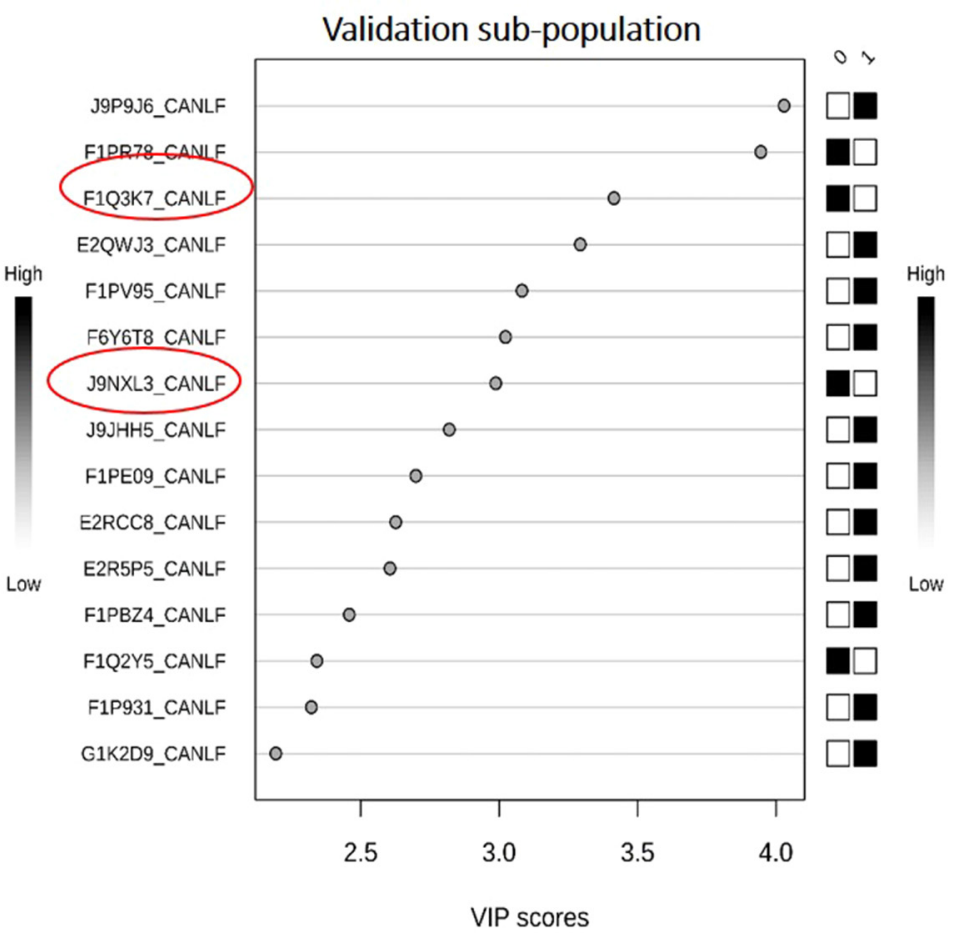

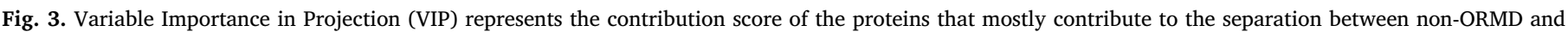

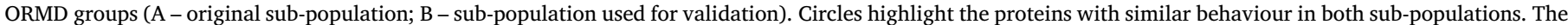
black and white boxes on the right indicate the relative concentrations of the corresponding protein in each group under study. 0 - non-ORMD; 1 -ORMD.

increased in the saliva of ORMD group of dogs. Superoxide dismutase is a major antioxidant enzyme. Al-Rawi [27] reported an increase in the levels of this enzyme in serum and saliva of diabetic persons. This increase was suggested to be due to the increase in free radical production and a pro-oxidant environment, in diabetes, with the reaction of the antioxidant defence system to counter balance it. In the case of obesity, this increase in the levels of superoxide dismutase appears to be only in initial states and when obesity persists there is a depletion of antioxidant sources, including this enzyme [28].

Glutathione $\mathrm{S}$ transferase belongs to a class of proteins involved in detoxification of endogenous and exogenous compounds. This enzyme, in concert with antioxidant systems modulate oxidative stress associated with diabetes mellitus. All of the animals tested in the present study (in both sub-populations) that were diagnosed with ORMD were hyperglycemic and the increase in the levels of this enzyme in their saliva goes in line with studies reporting higher serum levels of glutathione-S-transferase associated with increased levels of glycated haemoglobin [29]. Additionally, to the two proteins refereed before, the increase in the levels of Hsp70 also supports the thought of augmented oxidative stress in ORMD dogs. Heat shock proteins are endogenous highly conserved molecules produced by cells when exposed to a cell stressor. Increased levels of Hsp70 were observed to be increased in patients with diabetes mellitus in different studies [30,31].

Although protein S100 is involved in cytoskeletal dynamics, this protein can be released from cytoplasm in response to tissue/cell damage. $\$ 100$ proteins serve as danger signal and are crucial in regulating immune homeostasis and inflammation [32]. In same line, in previous proteomic-based studies in serum, altered immune system in dogs with ORMD was observed $[7,10]$.

The proteins glucose 6-phosphate isomerase and phosphoglycerate mutase participate in glycolysis and were observed to be augmented in the saliva of ORMD dogs. Glucose 6-phosphate isomerase catalyses the interconversion of glucose- 6 phosphate and fructose- 6 phosphate, whereas phosphoglycerate mutase catalyses the transfer of phosphate groups from 3-phosphoglycerate to 2-phosphoglycerate. The reason why these two enzymes were observed to be increased in the saliva of ORMD dogs needs further clarification. Although glycolysis appears as a potential route for lowering hyperinsulinemia, its occurrence appears to be different in type 1 and type 2 diabetes. Whereas in the first, insulin deficiency results in lowering glycolysis, in type 2 diabetes, hyperinsulinemia brings about a compensatory increase in rates of glycolysis in the liver, adipose tissue, and pancreatic $\beta$ cells [33], until tissues became resistant to insulin.

The levels of the enzyme glucose-6-phosphate dehydrogenase, involved in the pentose phosphate pathway, were already reported to be increased in diabetes [34]. The activity of this enzyme is related with the production of NADPH, being this last an important intracellular reductant, counteracting oxidative stress. It is possible that, at an initial phase of diabetes, increases in this enzyme prevent the oxidative stress known to associate to the development of diabetes and metabolic syndrome. Xu et al. [35] reported that chronic hyperglycemia causes inhibition of glucose-6-phosphate dehydrogenase, resulting in increased oxidative stress.

Through PLS-DA it was also observed the contribution of Can f6 allergen to separate the two groups (ORMD vs. Non ORMD), with this protein decreased in ORMD group. Can $\mathrm{f} 6$ is a dog allergen from the family of lipocalins. Lipocalins owe their name to the fact that they usually carry lipids (or other hydrophobic compounds). These are important allergens to humans, existent in dog saliva [36]. The amounts of lipocalin are usually increased in obesity [37].

The limitations of this study include a relatively low number of animals used, although it is in line with previously reported studies based on proteomic approach [24]. The need for a considerable number of animals was highlighted by the different results obtained for the two sub-populations of animals used in this study. It was necessary to increase the number of animals to access differences in salivary proteins that were not previously observed using only six animals per group. The studied animals were from different households with different diets, 
environmental conditions and care. Although this could contribute to increase variability, this had the advantage of permitting to evaluate a naturally occurring ORMD and get a true clinical picture. Furthermore, ideally a validation of the analytes of interest detected in this study would be desirable in a population of 200-300 dogs, with ORMD, to get an estimated power of $75 \%$.

\section{Conclusion}

In the present study, salivary proteomes of dogs with and without ORMD were compared and nine proteins with different abundance levels were identified, with two additional proteins observed (using PLSDA multivariate model) as contributing to separating groups. Among these, proteins involved in glycolysis, immune system and oxidative stress were increased in the saliva of ORMD animals.

Saliva is a fluid presenting variations among individual animals, breeds, through the day and with different stimulation. Moreover, metabolic syndrome includes different combinations of diseases. Even with these factors leading to variability in dogs with ORMD, the referred salivary proteins were observed to differ between groups. As such, the present study shows the potential of saliva to study metabolic diseases in dogs. However, further long-scale studies are needed to confirm these findings and to increase the knowledge about the possible associations between these proteins and ORMD in dogs.

\section{Acknowledgments}

Authors would like to acknowledge all the dog owners for the participation in the study.

\section{Funding}

This paper was funded by national funding through Portuguese Science Foundation (FCT) under the project UID/AGR/00115/2013 (ICAAM - University of Évora) and by a grant from the Robles Chillida Foundation through its support program for the promotion on research in Health Sciences. This work was also financed by the European Regional Development Fund (ERDF) through the COMPETE 2020 Operational Programme for Competitiveness and Internationalisation and Portuguese national funds via FCT, under projects: POCI-01-0145FEDER-029311; POCI-01-0145-FEDER-007440 (ref.; UID/NEU/04539/ 2013), POCI-01-0145-FEDER-016428 (ref.: SAICTPAC/0010/2015), and POCI-01-0145-FEDER-016795 (ref.: PTDC/NEU-SCC/7051/2014); and by The National Mass Spectrometry Network (RNEM) under the contract POCI-01-0145-FEDER-402-022125 (ref.: ROTEIRO/0028/ 2013). The authors also had financial support from FCT in the form of Elsa Lamy's FCT investigator contract IF/01778/2013. Asta Tvarijonaviciute was supported by the program "Juan de la Cierva Incorporacion" of 'Ministerio de Economia y Competitividad', Spain, through a postdoctoral grant. Funders had no role in study design, data collection, analysis, interpretation, or writing of the report.

\section{Appendix A. Supplementary data}

Supplementary data to this article can be found online at https:// doi.org/10.1016/j.jprot.2019.04.010.

\section{References}

[1] K.G.M.M. Alberti, R.H. Eckel, S.M. Grundy, P.Z. Zimmet, J.I. Cleeman, K.A. Donato, et al., Harmonizing the metabolic syndrome: a joint interim statement of the International Diabetes Federation Task Force on Epidemiology and Prevention; National Heart, Lung, and Blood Institute; American Heart Association; World Heart Federation; International Atherosclerosis Society; and International Association for the Study of Obesity, Circulation 120 (16) (2009 Oct 20) 1640-1645.

[2] R.K. Simmons, K.G.M.M. Alberti, E.A.M. Gale, S. Colagiuri, J. Tuomilehto, Q. Qiao, et al., The metabolic syndrome: useful concept or clinical tool? Report of a WHO
Expert Consultation, Diabetologia 53 (4) (2010 Apr) 600-605.

[3] E.M. Lund, P.J. Armstrong, C.A. Kirk, J.S. Klausner, Prevalence and risk factors for obesity in adult dogs from private US veterinary practices, Int. J. Appl. Res. Vet. Med. 4 (2) (2006) 177.

[4] K.R. Verkest, L.M. Fleeman, J.M. Morton, K. Ishioka, J.S. Rand, Compensation for obesity-induced insulin resistance in dogs: assessment of the effects of leptin, adiponectin, and glucagon-like peptide-1 using path analysis, Domest. Anim. Endocrinol. 41 (1) (2011 Jul 1) 24-34.

[5] A.R. Bodey, A.R. Michell, Epidemiological study of blood Sressure in omestic dogs, J. Small Anim. Pract. 37 (3) (1996 Mar 1) 116-125.

[6] T. Chikamune, H. Katamoto, F. Ohashi, Y. Shimada, Serum lipid and lipoprotein concentrations in obese dogs, J. Vet. Med. Sci. 57 (4) (1995 Aug 15) 595-598.

[7] A. Tvarijonaviciute, R. Barić-Rafaj, A. Horvatic, A. Muñoz-Prieto, N. Guillemin, E. Lamy, A. Tumpa, J.J. Ceron, S. Martinez-Subiela, V. Mrljak, Identification of changes in serum analytes and possible metabolic pathways associated with canine obesity-related metabolic dysfunction, Vet. J. 244 (2019) 51-59.

[8] K.R. Verkest, Is the metabolic syndrome a useful clinical concept in dogs? A review of the evidence, Vet. J. 199 (1) (2014 Jan) 24-30.

[9] A. Tvarijonaviciute, J.J. Ceron, S.L. Holden, D.J. Cuthbertson, V. Biourge, P.J. Morris, et al., Obesity-related metabolic dysfunction in dogs: a comparison with human metabolic syndrome, BMC Vet. Res. 8 (2012 Aug 28) 147.

[10] A. Tvarijonaviciute, J.J. Ceron, C. de Torre, B.B. Ljubić, S.L. Holden, Y. Queau, et al., Obese dogs with and without obesity-related metabolic dysfunction - a proteomic approach, BMC Vet. Res. [Internet] 12 (2016 Sep 20) Available from https://www.ncbi.nlm.nih.gov/pmc/articles/PMC5028949/.

[11] D. Piantedosi, A. Di Loria, J. Guccione, A. De Rosa, S. Fabbri, L. Cortese, et al., Serum biochemistry profile, inflammatory cytokines, adipokines and cardiovascular findings in obese dogs, Vet. J. 216 (2016 Oct) 72-78.

[12] M. Castagnola, E. Scarano, G.C. Passali, I. Messana, T. Cabras, F. Iavarone, et al., Salivary biomarkers and proteomics: future diagnostic and clinical utilities, Acta Otorhinolaryngol. Ital. 37 (2) (2017 Apr) 94-101.

[13] P. Shi, J.M. Goodson, M.-L. Hartman, H. Hasturk, T. Yaskell, J. Vargas, et al., Continuous metabolic syndrome scores for children using salivary biomarkers, PLoS ONE [Internet]. 10 (9) (2015 Sep 29) Available from: https://www.ncbi.nlm.nih. gov/pmc/articles/PMC4587796/.

[14] J.M. Goodson, A. Kantarci, M.-L. Hartman, G.V. Denis, D. Stephens, H. Hasturk, et al., Metabolic disease risk in children by salivary biomarker analysis, PLoS ONE [Internet]. 9 (6) (2014 Jun 10) Available from https://www.ncbi.nlm.nih.gov/ pmc/articles/PMC4051609/.

[15] A. Chauhan, S.S. Yadav, P. Dwivedi, N. Lal, K. Usman, S. Khattri, Correlation of serum and salivary cytokines level with clinical parameters in metabolic syndrome with periodontitis, J. Clin. Lab. Anal. 30 (5) (2016 Sep 1) 649-655.

[16] D. Laflamme, Nutritional management, Vet. Clin. North Am. Small Anim. Pract. 27 (6) (1997 Nov) 1561-1577.

[17] A. Tvarijonaviciute, J.D. Carrillo-Sanchez, J.D. García-Martinez, F. Tecles, S. Martinez-Subiela, A.J. German, et al., Measurement of salivary adiponectin concentrations in dogs, Vet. Clin. Pathol. 43 (3) (2014).

[18] Short GeLC-SWATH: A fast and reliable quantitative approach for proteomic screenings - Anjo - 2015 - Proteomics - Wiley Online Library [Internet]. [cited 2018 Mar 1]. 18 https://doi.org/10.1002/pmic.201400221/abstract;jsessionid= 858AF2C17EC8FA92F5E8969AD91BAEE0.f04t01

[19] L.C. Gillet, P. Navarro, S. Tate, H. Röst, N. Selevsek, L. Reiter, et al., Targeted data extraction of the MS/MS Spectra generated by data-independent acquisition: a new concept for consistent and accurate proteome analysis, Mol. Cell Proteomics MCP [Internet] 11 (6) (2012 Jun) Available from: https://www.ncbi.nlm.nih.gov/pmc/ articles/PMC3433915/.

[20] L. Sennels, J.-C. Bukowski-Wills, J. Rappsilber, Improved results in proteomics by use of local and peptide-class specific false discovery rates, BMC Bioinforma. 10 (2009 Jun 12) 179.

[21] W.H. Tang, I.V. Shilov, S.L. Seymour, Nonlinear fitting method for determining local false discovery rates from decoy database searches, J. Proteome Res. 7 (9) (2008 Sep) 3661-3667.

[22] J. Xia, D.S. Wishart, Using metaboanalyst 3.0 for comprehensive metabolomics data analysis: using metaboanalyst 3.0 for comprehensive metabolomics data analysis, in: A. Bateman, W.R. Pearson, L.D. Stein, G.D. Stormo, J.R. Yates (Eds.), Current Protocols in Bioinformatics [Internet], John Wiley \& Sons, Inc., Hoboken, NJ, USA, 2016, , https://doi.org/10.1002/cpbi.11 [cited 2018 Jan 17]. p. 14.10.1-14.10.91. Available from:.

[23] S.M.F. Torres, E. Furrow, C.P. Souza, J.L. Granick, E.P. de Jong, T.J. Griffin, et al., Salivary proteomics of healthy dogs: an in depth catalog, Guo X, editor, PLoS ONE 13 (1) (2018 Jan 12) e0191307.

[24] S. Pasha, T. Inui, I. Chapple, S. Harris, L. Holcombe, M.M. Grant, The saliva proteome of dogs: variations within and between breeds and between species, Proteomics (2018 Jan 12) 1700293.

[25] S. Lucena, A.V. Coelho, F. Capela-Silva, A. Tvarijonaviciute, E. Lamy, The effect of breed, gender, and acid stimulation in dog saliva proteome, Bio. Med. Res. Int. (2018) 1-12 2018 Jun 3.

[26] C.K. Roberts, K.K. Sindhu, Oxidative stress and metabolic syndrome, Life Sci. 84 (21-22) (2009 May 22) 705-712.

[27] N.H. Al-Rawi, Oxidative stress, antioxidant status and lipid profile in the saliva of type 2 diabetics, Diab. Vasc. Dis. Res. 8 (1) (2011 Jan) 22-28.

[28] A. Fernández-Sánchez, E. Madrigal-Santillán, M. Bautista, J. Esquivel-Soto, A. Morales-González, C. Esquivel-Chirino, I. Durante-Montiel, G. Sánchez-Rivera, C. Valadez-Vega, J.A. Morales-González, Inflammation, oxidative stress, and obesity, Int. J. Mol. Sci. 12 (5) (2011) 3117-3132.

[29] S.U. Velladath, A. Das, Kumar RKN, Erythrocyte glutathione-S-transferase activity 
in diabetics and its association with HBA1c, Webmed Central Clin. Biochem. 2 (7) (2011) WMC002004.

[30] M. Nakhjavani, A. Morteza, A.A. Nargesi, E. Mostafavi, A. Esteghamati, Appearance of leptin-HSP70 correlation, in type 2 diabetes, Meta Gene. 1 (2013 Oct 8) 1-7.

[31] F. Giacco, M. Brownlee, Oxidative stress and diabetic complications, Circ. Res. 107 (9) (2010 Oct 29) 1058-1070.

[32] L. Bao, A.F. Odell, S.L. Stephen, S.B. Wheatcroft, J.H. Walker, S. Ponnambalam, The S100A6 calcium-binding protein regulates endothelial cell-cycle progression and senescence, FEBS J. 279 (24) (2012 Dec) 4576-4588.

[33] X. Guo, H. Li, H. Xu, S. Woo, H. Dong, F. Lu, A.J. Lange, C. Wu, Glycolysis in the control of blood glucose homeostasis, Acta Pharm. Sin. B 2 (4) (2012) 358-367.

[34] S.A. Hamzah, L.A. Hamza and H.S. Rahman, Impact of diabetes mellitus type 2 in the activity of glucose-6-phosphate dehydrogenase in human erythrocyte, Kurdistan J. Appl. Res. 3 (1) (2018) 58-62.

[35] Y. Xu, B.W. Osborne, R.C. Stanton, Diabetes causes inhibition of glucose-6-phosphate dehydrogenase via activation of PKA, which contributes to oxidative stress in rat kidney cortex, Am. J. Physiol. Ren. Physiol. 289 (5) (2005 Nov) F1040-F1047.

[36] E. Jensen-Jarolim, L.F. Pacios, R. Bianchini, G. Hofstetter, F. Roth-Walter, Structural similarities of human and mammalian lipocalins, and their function in innate immunity and allergy, Allergy 71 (3) (2016 Mar) 286-294.

[37] I.K. Law, A. Xu, K.S. Lam, T. Berger, T.W. Mak, P.M. Vanhoutte, J.T. Liu, G. Sweeney, M. Zhou, B. Yang, Y. Wang, Lipocalin-2 deficiency attenuates insulin resistance associated with aging and obesity, Diabetes 59 (4) (2010 Apr) 872-882. 